\title{
Selection at two sex-linked loci
}

\author{
Robin E. Owen
}

Department of Biological Sciences, University of Calgary, Calgary, Alberta T2N 1N4, Canada.

Recurrence equations are derived for selection at two sex-linked or haplodiploid loci, each with two alleles. Equilibrium equations containing only the female gametic frequencies are obtained by using a transformation that absorbs the male gametic frequencies. It is shown that gametic equilibria occur at a stationary point of the geometric mean viability if, and only if, $\frac{1}{2} r_{f}\left[x_{1} x_{4}\left(v_{11}+v_{44}\right)-x_{2} x_{3}\left(v_{22}+v_{33}\right)\right] w_{22}=0$, where $r_{f}$ is the recombination fraction in females, $w_{22}$ is the viability of the double heterozygote, $x_{1}, x_{2}, x_{3}, x_{4}$ are the frequencies of the female gametes $A B$, $A b, a B, a b$ respectively and $v_{11}, v_{22}, v_{33}, v_{44}$ are the corresponding male viabilities. Therefore, in general, linkage equilibrium is neither sufficient nor necessary for the mean viability to be at a stationary point and there will always be some linkage disequilibrium in the system, either in females or males or both. Symmetrical selection in females is analysed in some detail and it is shown that unless there is also a particular type of symmetrical selection in males then only asymmetrical equilibria can occur. The hitchhiking effect at sex-linked and autosomal loci is compared, and it is found that with no recombination in males at autosomal loci the effect can be stronger than at sex-linked loci.

\section{INTRODUCTION}

The theory of selection at two autosomal loci has received considerable attention and significant progress has been made on specific selection models (Ewens, 1979). The properties of the system with general viability parameters are less well understood (Karlin, 1975), although it is possible to specify the maximum number of equilibria that can occur (Arunachalam and Owen, 1971; Karlin, 1975) and expressions have been derived for the change in mean fitness (Arunachalam and Owen, 1971; Nagalaki, 1977).

Two-locus haploid selection models have also been analysed (Nei, 1967; Feldman, 1971) revealing, essentially, that no polymorphic equilibria can be stable.

In contrast no work has been done on selection at two sex-linked (haplodiploid) loci even though theory for a single locus with two or multiple alleles is quite well developed (Edwards, 1977). Indeed little consideration has been given to two sexlinked loci at all. Wright (1933) derived an expression for the rate of approach to linkage equilibrium using the method of path coefficients, while Bennett (1963) derived the same formula directly from the gametic recurrence equations, concluding that the rate is approximately twothirds that of a pair of corresponding autosomal loci. Owen (1980) analysed a situation application to the social Hymenoptera where a proportion of the males are produced by the workers and found that this had, at most, a marginal effect on the rate of approach to equilibrium. Clegg and Cavener (1982) experimentally examined the rate of decay of linkage disequilibrium in populations of Drosophila melanogaster with both discrete and overlapping generations, and derived a formula for the rate of approach to equilibrium with overlapping generations.

In this paper some models of selection at two sex-linked or haplodiploid loci are analysed. The recurrence equations with general viabilities are derived and equilibrium equations containing only the female gametic frequencies are obtained, by using a transformation that absorbs the male gametic frequencies. Some general properties of the system are examined and an interesting result obtained that linkage equilibrium, either in females or males, is in general neither a sufficient nor necessary condition for the geometric mean viability to be at a stationary point when the population is at gametic equilibrium. 
Symmetrical selection in females is analysed with symmetrical or asymmetrical selection in males and it is shown that unless there is a particular balance of selection coefficients in the males then asymmetrical equilibria are bound to result.

Sex-linked or haplodiploid loci are predicted to have lower levels of genetic variability than autosomal loci whether selection or mutation-drift maintain variability (Avery, 1984). The role of hitchhiking has been discussed (Lester and Selander, 1979; Pamilo and Crozier, 1981) but remains unclear. The hitchhiking effect at sex-linked and autosomal loci is compared, and it is shown that if there is no recombination in males at autosomal loci (e.g., as in Drosophila) then the effect can be stronger than at sex-linked loci.

\section{SELECTION: GENERAL MODEL}

\section{Recurrence equations}

Assume two sex-linked loci each with two alleles, $A, a$ and $B, b$ respectively. Let the population be censused at the gametic stage, then at time $t$ the frequencies of the gametes are;

\section{$A B \quad A b \quad a B \quad a b$}

Females: $\quad x_{1, t} \quad x_{2, t} \quad x_{3, t} \quad x_{4, t}$

Males: $\quad y_{1, t} \quad y_{2, t} \quad y_{3, t} \quad y_{4, t}$

where

$$
\sum_{i=1}^{4} x_{i, t}=1 \quad \text { and } \quad \sum_{i=1}^{4} y_{i, t}=1
$$

An infinite population size and random union of gametes are also assumed. Let the female and male viability matrices $W$ and $V$ respectively be,

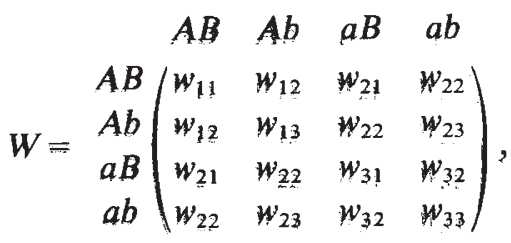

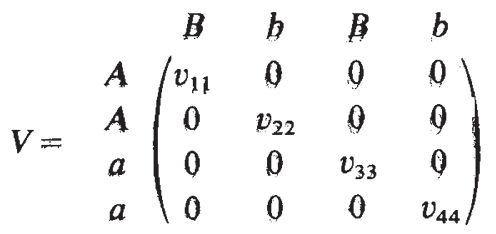

The female viability matrix is the familiar one, assuming the coupling and repulsion double heterozygotes to have the same fitness (Ewens, 1979). The male viabilities are given in the form $v_{11}$, etc. to distinguish them from single locus viabilities $\left(v_{1}\right.$, etc.) and the matrix is diagonal to be consistent with the single locus multiallelic formulation (Cannings, 1969a). If the recombination fraction in females is $r_{f}$, and remembering that there is no recombination in males, then the recurrence equations are seen to be;

Females:

$$
\begin{aligned}
\bar{w} x_{1, t+1}= & x_{1, t} y_{1, t} w_{11} \\
& +\frac{1}{2}\left(x_{1, t} y_{2, t}+x_{2, t} y_{1, t}\right) w_{12} \\
& +\frac{1}{2}\left(x_{1, t} y_{3, t}+x_{3, t} y_{1, t}\right) w_{21} \\
& +\frac{1}{2}\left(x_{1, t} y_{4, t}+y_{1, t} x_{4, t}\right) w_{22} \\
& -\frac{1}{2} r_{\mathrm{f}} I w_{22} \\
\bar{w} x_{2, t+1}= & x_{2, t} y_{2, t} w_{13} \\
& +\frac{1}{2}\left(x_{1, t} y_{2, t}+x_{2, t} y_{1, t}\right) w_{12} \\
& +\frac{1}{2}\left(x_{2, t} y_{4, t}+x_{4, t} y_{2, t}\right) w_{23} \\
& +\frac{1}{2}\left(x_{2, t} y_{3, t}+x_{3, t} y_{2, t}\right) w_{22} \\
& +\frac{1}{2} r_{\mathrm{f}} I w_{22} \\
= & x_{3, t} y_{3, t} w_{31} \\
& +\frac{1}{2}\left(x_{1, t} y_{3, t}+x_{3, t} y_{1, t}\right) w_{21} \\
& +\frac{1}{2}\left(x_{3, t} y_{4, t}+x_{4, t} y_{3, t}\right) w_{32} \\
& +\frac{1}{2}\left(x_{2, t} y_{3, t}+x_{3, t} y_{2, t}\right) w_{22} \\
& +\frac{1}{2} r_{\mathrm{f}} I w_{22} \\
\bar{w} x_{3, t} & x_{4, t} y_{4, t} w_{33} \\
& +\frac{1}{2}\left(x_{3, t} y_{4, t}+x_{4, t} y_{3, t}\right) w_{32} \\
& +\frac{1}{2}\left(x_{2, t} y_{4, t}+x_{4, t} y_{2, t}\right) w_{23} \\
& +\frac{1}{2}\left(x_{1, t} y_{4, t}+y_{1, t} x_{4, t}\right) w_{22} \\
& -\frac{1}{2} r_{\mathrm{f}} I w_{22} \\
\bar{w} x_{4, t} &
\end{aligned}
$$

where

$$
I=x_{1, t} y_{4, t}+x_{4, t} y_{1, t}-x_{2, t} y_{3, t}-x_{3, t} y_{2, t}
$$

and $\bar{w}$ is the mean viability of the females.

Males:

$$
\bar{v} y_{i, t+1}=x_{i, t} v_{i i}, \quad i=1,2,3,4
$$

where $\vec{v}$ is the mean viability of the males. Before selection the genotypic frequencies of the males are the same as the gametic frequencies of the female parents, hence after selection $y_{i, t}=$ $x_{i, t-1} v_{i i} / \vec{v}$, therefore the $y$ 's in equations (1) can be eliminated by making this substitution. Next define 
the matrix of compound viabilities $U=$ $\frac{1}{2}(W V+V W)$. At equilibrium $x_{i, t-1}=x_{i, t}=x_{i, t+1}=x_{i}$ (say), therefore the female recurrence equations become, in terms of the compound viabilities,

$$
\begin{aligned}
\hat{M} x_{i} & =x_{i} u_{i}+\eta_{i} r_{\mathrm{f}}\left(x_{1} x_{4} u_{14}-x_{2} x_{3} u_{23}\right), \\
i & =1,2,3,4
\end{aligned}
$$

with $\eta_{1}=\eta_{4}=-1, \eta_{2}=\eta_{3}=1$, and where the marginal fitness of gamete $i$ is defined as $u_{i}=\sum_{j}^{4} x_{j} u_{i j}$. The geometric mean viability of the entire population

$$
M=\bar{w} \bar{v} .
$$

(In equations (3) the circumflex indicates the equilibrium value of $M$ ). The transformation employed above is analogous to that used by $\mathrm{Li}$ (1967) for a single locus with two alleles. The female equilibrium gametic frequencies are given by the solution of equations (3), and the male equilibrium frequencies are obtained by substitution in equations (2). At two sex-linked loci there are two coefficients of linkage disequilibrium, that for females $D_{\mathrm{f}}=x_{1, t} x_{4, t}-x_{2, t} x_{3, t}$ and that for males $D_{\mathrm{m}}=y_{1, t} y_{4, t}-y_{2, t} y_{3, t}$.

Equations (3) are of identical form to those for autosomal loci and, consequently, like the autosomal case relatively little can be said about the general properties of the system (Edwards, 1977), however some conclusions can be drawn.

\section{Stationary point of the mean viability}

The mean viability $\hat{M}$ is the same function as that obtained in the case of a single locus with four alleles with gene frequencies $x_{1}, x_{2}, x_{3}, x_{4}$ in females.

The internal equilibrium values of the female gene frequencies occur at the stationary point of the viability matrix $\frac{1}{2}(W V+V W)$ as proved by Cannings $(1968,1969 a)$. Thus the mean viability of the four-allele system can be seen to be the same as equation (4). This has internal stationary point(s) at $x_{1}^{*}, x_{2}^{*}, x_{3}^{*}, x_{4}^{*}$, given by,

$$
M^{*}=u_{i}^{*}, \quad i=1 \ldots 4
$$

where $u_{i}^{*}=\sum_{j}^{4} x_{j}^{*} u_{i j}$, and the equilibrium mean viability is written as $M^{*}$. Assuming an equilibrium exists at $x_{i}^{*}=x_{i}$, then substitution into equations (3) yields,

$$
\begin{aligned}
\hat{M} x_{i} & =M^{*} x_{i}+\eta_{i} r_{\mathrm{f}}\left(x_{1} x_{4} u_{14}-x_{2} x_{3} u_{23}\right), \\
i & =1 \ldots 4
\end{aligned}
$$

Hence gametic equilibrium occurs at a stationary point of the mean viability if and only if $r_{\mathrm{f}}\left(x_{1} x_{4} u_{14}-x_{2} x_{3} u_{23}\right)=0$, i.e., if

$$
\frac{1}{2} r_{\mathrm{fL}}\left[x_{1} x_{4}\left(v_{11}+v_{44}\right)-x_{2} x_{3}\left(v_{22}+v_{33}\right)\right] w_{22}=0 .
$$

The well-known analogous condition for two autosomal loci that $r D W_{22}=0$, was proved by Moran (1964), and the proof given above follows that of Edwards (1977). Clearly the mean viability can only be maximized at equilibrium when either (a) $r_{f}=0, \quad$ (b) $\quad w_{22}=0, \quad$ (c) $x_{1} x_{4}\left(v_{11}+v_{44}\right)-$ $x_{2} x_{3}\left(v_{22}+v_{33}\right)=0$. Therefore for two-sex-linked loci the condition that linkage equilibrium must hold at equilibrium for the mean viability to be maximized or even be at a stationary point, is, in general, neither sufficient nor necessary. If, however, a particular relationship holds between the male viabilities then $\hat{D}_{\mathrm{f}}=0$ is a sufficient condition. Let $\left(v_{11}+v_{44}\right)=\left(v_{22}+v_{33}\right)=v_{\mathrm{m}}$, then equation (7) becomes $\frac{1}{2} r_{\mathrm{f}}\left(x_{1} x_{4}-x_{2} x_{3}\right) v_{\mathrm{m}} w_{22}=0$ hence $\hat{D}_{\mathrm{f}}=0$ is sufficient, however it also follows that $\hat{D}_{\mathrm{m}} \neq 0$ in this case. In fact as long as there is selection in males then $\hat{D}_{\mathrm{f}}, \hat{D}_{\mathrm{m}}$ and $\hat{I}$ cannot all equal zero at equilibrium, whether or not the mean viability is maximized.

\section{No Selection in Males}

If there are no selective differences between male genotypes $\left(v_{11}=v_{22}=v_{33}=v_{44}=1\right)$ then the equilibrium equations (3) reduce to the standard autosomal ones with $r_{\mathrm{f}}$ replacing $r$, and in this case $\hat{D}_{\mathrm{m}}=\hat{D}_{\mathrm{f}}$ and $\hat{I}=2 \hat{D}_{\mathrm{f}}$. Thus the situation is equivalent to selection at two corresponding autosomal loci with half the recombination fraction.

\section{No selection in females}

If there are no selective differences between female genotypes then selection acts only on the haploid males. In this case equations (2) at equilibrium reduce to the two locus haploid equations at equilibrium (Feldman, 1971), and for the general viability model it seems fairly clear that at most one internal equilibrium can exist and this is unstable (Feldman, 1971). Therefore it seems likely that the gamete (male genotype) with the greatest viability will proceed to fixation, as in the single-locus four allele case (Cannings, 1969a).

\section{Epistasis}

It is interesting to note what form epistatic interactions take at sex-linked loci. Define as the components of epistatic interactions of compound 
viabilities the sums,

$$
\begin{aligned}
& \varepsilon_{1}=u_{11}-u_{12}-u_{13}+u_{14} \\
& \varepsilon_{2}=u_{12}-u_{22}-u_{32}+u_{42} \\
& \varepsilon_{3}=u_{13}-u_{23}-u_{33}+u_{43} \\
& \varepsilon_{4}=u_{14}-u_{24}-u_{34}+u_{44}
\end{aligned}
$$

which are comparable to the epistatic interactions in the two-locus autosomal case (cf. Lewontin and Kojima, 1960; Bodmer and Felsenstein, 1967). Dividing each of equations (3) by the corresponding $x_{i}$ and appropriately changing signs and adding yields,

$$
\varepsilon_{1} x_{1}+\varepsilon_{2} x_{2}+\varepsilon_{3} x_{3}+\varepsilon_{4} x_{4}-\frac{1}{2} r_{\mathrm{f}} w_{22} I \sum_{i=1}^{4} \frac{1}{x_{i}}=0 .
$$

Therefore

$$
\frac{1}{2} r_{\mathrm{f}} w_{22} I=\left(\sum_{i=1}^{4} \varepsilon_{i} x_{i}\right) / \sum_{i=1}^{4} \frac{1}{x_{i}} .
$$

The sum $\sum \varepsilon_{i} x_{i}$ may be called the "average epistatic interaction" (of the compounded viabilities), and clearly $I=0$ in the case of additive gene effects. By the Cauchy-Schwartz inequality,

$$
\left(\sum_{i=1}^{4} \varepsilon_{i} x_{i}\right)^{2} \leqq\left(\sum_{i=1}^{4} \varepsilon_{i}^{2}\right)\left(\sum_{i=1}^{4} x_{i}^{2}\right) \leqq \sum_{i=1}^{4} \varepsilon_{i}^{2} .
$$

Therefore

$$
\left|\sum_{i=1}^{4} \varepsilon_{i} x_{i}\right| \leqq \sqrt{\sum_{i=1}^{4} \varepsilon_{i}^{2}} .
$$

The latter term may be called the "root mean square epistatic interaction", or the r.m.s. interaction for short.

Also $1 /\left(\frac{1}{4} \sum_{i=1}^{4} 1 / x_{i}\right)$ is the harmonic mean of $x_{1}, x_{2}, x_{3}, x_{4}$ and is therefore not greater than the arithmetic mean $\left(x_{1}+x_{2}+x_{3}+x_{4}\right) / 4=\frac{1}{4}$. Thus

$$
4 /\left(\sum_{i=1}^{4} \frac{1}{x_{i}}\right) \leqq \frac{1}{4} \quad \text { and } \quad \sum_{i=1}^{4} \frac{1}{x_{i}} \geqq 16 .
$$

It follows that $\frac{1}{2} r_{\mathrm{f}} w_{22}|I| \leqq($ r.m.s. interaction $) / 16$ i.e., $|I| \leqq$ (r.m.s. interaction $) / 8 r_{\mathrm{f}} w_{22}$.

This differs from the corresponding result of Arunachalam and Owen (1970) by a factor of two which arises from the haplodiploid situation vis-àvis the diploid one.

\section{SYMMETRICAL SELECTION IN FEMALES}

One specific selection model to receive considerable attention is the symmetric viability model (e.g., Kimura, 1956; Lewontin and Kojima, 1960;
Bodmer and Felsenstein, 1967; Karlin and Feldman, 1970), and it has been found that both symmetric $\left(x_{1}=x_{4}, x_{2}=x_{3}\right)$ and asymmetric (e.g., $x_{1} \neq$ $x_{4}, x_{2}=x_{3}$ ) equilibria can occur with selection of this type (Karlin and Feldman, 1970).

In this section a model of symmetrical selection in females is analysed for some sub-models of symmetrical and asymmetrical selection in males. The female viabilities are designated thus,

$$
\left(\begin{array}{ccc}
1-\delta_{\mathrm{f}} & 1-\beta & 1-\alpha_{\mathrm{f}} \\
1-\gamma & 1 & 1-\gamma \\
1-\alpha_{\mathrm{f}} & 1-\beta & 1-\delta_{\mathrm{f}}
\end{array}\right)
$$

\section{SYMMETRICAL SELECTION IN MALES}

Two complementary sub-models will be considered.

\section{(1) Male viabilities}

$$
v_{11}=v_{44}=1-\delta_{\mathrm{m}}, \quad v_{22}=v_{33}=1 .
$$

\section{Symmetrical equilibria}

Substitution of the above male viabilities and female viabilities (8) into equations (3) and appropriate subtraction gives,

$$
\begin{aligned}
\hat{M}\left(x_{1}-x_{4}\right)= & \left(x_{1}^{2}-x_{4}^{2}\right)\left(1-\delta_{\mathrm{f}}\right)\left(1-\delta_{\mathrm{m}}\right) \\
& +\frac{1}{2}\left(x_{1} x_{2}-x_{3} x_{4}\right)(1-\beta)\left(2-\delta_{\mathrm{m}}\right) \\
& +\frac{1}{2}\left(x_{1} x_{3}-x_{2} x_{4}\right)(1-\gamma)\left(2-\delta_{\mathrm{m}}\right) \\
\hat{M}\left(x_{2}-x_{3}\right)= & \left(x_{2}^{2}-x_{3}^{2}\right)\left(1-\alpha_{\mathrm{f}}\right) \\
& +\frac{1}{2}\left(x_{1} x_{2}-x_{3} x_{4}\right)(1-\beta)\left(2-\delta_{\mathrm{m}}\right) \\
& -\frac{1}{2}\left(x_{1} x_{3}-x_{2} x_{4}\right)(1-\gamma)\left(2-\delta_{\mathrm{m}}\right) .
\end{aligned}
$$

Clearly, as in the autosomal case, $x_{1}=x_{4}$, and $x_{2}=x_{3}$, is a solution of the two simultaneous equations (9a) and (9b). Therefore substitution of $x_{1}=x_{4}=\frac{1}{4}+D_{\mathrm{f}}$ and $x_{2}=x_{3}=\frac{1}{4}-D_{\mathrm{f}}$ into any one of equations (3) with symmetrical viabilities gives a cubic equation for the value of $D_{\mathrm{f}}$ at equilibrium,

$$
\begin{gathered}
64 k D_{\mathrm{f}}^{3}-16\left[m+2 \delta_{\mathrm{m}}\left(1+r_{\mathrm{f}}\right)\right] D_{\mathrm{f}}^{2} \\
-4\left[k-4 r_{\mathrm{f}}\left(2-\delta_{\mathrm{m}}\right)\right] D_{\mathrm{f}} \\
+m+2 \delta_{\mathrm{m}}\left(1-r_{\mathrm{f}}\right)=0
\end{gathered}
$$

where

$$
k=\left(2-\delta_{\mathrm{m}}\right)(\beta+\gamma)-\left[\alpha_{\mathrm{f}}+\delta_{\mathrm{f}}\left(1-\delta_{\mathrm{m}}\right)\right]
$$

and

$$
m=\delta_{\mathrm{f}}\left(1-\delta_{\mathrm{m}}\right)-\alpha_{\mathrm{f}} .
$$


Note that this equation for $D_{\mathrm{f}}$ is of the same degree (cubic) as the standard autosomal equation and reduces to it in the absence of selection in males (Bodmer and Felsenstein, 1967, equation 17) with, of course, $r_{\mathrm{f}}$ replacing $r$ and $D_{\mathrm{f}}$ replacing $D$. In this case however $D_{\mathrm{m}}=D_{\mathrm{f}}$. Nevertheless even with the rather simple symmetrical selection in males the terms in the equation are somewhat more complicated than in the autosomal case.

Some special cases will now be examined which allow equation (10) to be solved easily. These are analogous to the special cases analysed for autosomal loci (Bodmer and Felsenstein, 1967).

(1) $r_{\mathrm{f}}=0$; this corresponds to selection at a singlelocus with four alleles. Equation (10) reduces to,

$$
\left(16 D_{\mathrm{f}}^{2}-1\right)\left[4 k D_{\mathrm{f}}-\left(m+2 \delta_{\mathrm{m}}\right)\right]=0
$$

with solutions $D_{\mathrm{f}}=\frac{1}{4},-\frac{1}{4}$ and $\left(m+2 \delta_{\mathrm{m}}\right) / 4 k$

(2) $m+2 \delta_{\mathrm{m}}\left(1-r_{\mathrm{f}}\right)=0$; equation (10) reduces to

$$
\begin{gathered}
D_{\mathrm{f}}\left\{64 k D_{\mathrm{f}}^{2}-16\left[m+2 \delta_{\mathrm{m}}\left(1+r_{\mathrm{f}}\right)\right] D_{\mathrm{f}}\right. \\
\left.-4\left[k-4 r_{\mathrm{f}}\left(2-\delta_{\mathrm{m}}\right)\right]\right\}=0 .
\end{gathered}
$$

Therefore the condition for $D_{\mathrm{f}}=0$ is that

$$
\delta_{\mathrm{m}}=\left(\alpha_{\mathrm{f}}-\delta_{\mathrm{f}}\right) /\left[2\left(1-r_{\mathrm{f}}\right)-\delta_{\mathrm{m}}\right]
$$

or

$$
r_{\mathrm{f}}=\left[\delta_{\delta}-\alpha_{\mathrm{f}}+\delta_{\mathrm{m}}\left(2-\delta_{\mathrm{f}}\right)\right] / 2 \delta_{\mathrm{m}} .
$$

This is analogous to the autosomal case $\delta=\alpha$ considered by Lewontin and Kojima (1960), but at sex-linked loci the recombination fraction in females also enters into the conditions for $D_{\mathrm{f}}=0$.

Example: Let $\alpha_{\mathrm{f}}=0 \cdot 30, \beta=0 \cdot 15, \gamma=0 \cdot 10, \delta_{\mathrm{f}}=$ $0.20, \delta_{\mathrm{m}}=0.08, r_{\mathrm{f}}=0.275$; then $x_{1}=x_{2}=x_{3}=$ $x_{4}=0 \cdot 2500$ and $D_{\mathrm{f}}=0$. But of course, $y_{1}=y_{4}=$ $0 \cdot 2396, y_{2}=y_{3}=0 \cdot 2604, D_{\mathrm{m}}=-0.0104$ and $I=$ $-0 \cdot 0104$.

(3) $k=0$; if $(\beta+\gamma)\left(2-\delta_{\mathrm{m}}\right)=\alpha_{\mathrm{f}}+\delta_{\mathrm{f}}\left(1-\delta_{\mathrm{m}}\right)$ then equation (10) becomes a quadratic,

$$
\begin{aligned}
16\left[m+\delta_{\mathrm{m}}\left(1+r_{\mathrm{f}}\right)\right] D_{\mathrm{f}}^{2} & -16\left(2-\delta_{\mathrm{m}}\right) r_{\mathrm{f}} D_{\mathrm{f}}-m \\
- & -2 \delta_{\mathrm{m}}\left(1-r_{\mathrm{f}}\right)=0
\end{aligned}
$$

with solutions,

$$
\begin{aligned}
D_{\mathrm{f}}= & \frac{\left(1-\frac{1}{2} \delta_{\mathrm{m}}\right) r_{\mathrm{f}}}{m+2 \delta_{\mathrm{m}}\left(1+r_{\mathrm{f}}\right)} \\
& \pm \frac{1}{4}\left(\frac{16 r_{\mathrm{f}}^{2}+m^{2}+4 \delta_{\mathrm{m}}\left(m+\delta_{\mathrm{m}}-4 r_{\mathrm{f}}^{2}\right)}{m^{2}+4 \delta_{\mathrm{m}}\left[m\left(1+r_{\mathrm{f}}\right)+\delta_{\mathrm{m}}\left(1+r_{\mathrm{f}}\right)^{2}\right]}\right)^{1 / 2}
\end{aligned}
$$

When $\delta_{\mathrm{m}}=0$ equation (13) reduces to

$$
D_{\mathrm{f}}=r_{\mathrm{f}} / m \pm \frac{1}{4}\left(1+16 r_{\mathrm{f}}^{2} / m^{2}\right)^{1 / 2}
$$

which is equivalent to the autosomal case with $2(\beta+\gamma)=\alpha+\delta \quad$ (Bodmer and Felsenstein, 1967), and $r_{\mathrm{f}}$ replacing $r$.

Example: Let $\alpha_{\mathrm{f}}=0 \cdot 21, \beta=0 \cdot 15, \gamma=0 \cdot 10, \delta_{\mathrm{f}}=$ $0 \cdot 30, \delta_{\mathrm{m}}=0 \cdot 20, r_{\mathrm{f}}=0 \cdot 20$; then $k=0$ and $x_{1}=$ $x_{4}=0.194, \quad x_{2}=x_{3}=0.306$ with $D_{\mathrm{f}}=0.056$, also $y_{1}=y_{4}=0.168, \quad y_{2}=y_{3}=0.332, \quad D_{\mathrm{m}}=$ -0.082 and $I=-0 \cdot 138$.

\section{Asymmetrical equilibria}

Karlin and Feldman (1970) showed that asymmetric equilibria of the form $x_{1} \neq x_{4}, x_{2}=x_{3}$ (or vice versa) could exist at autosomal loci under the symmetric viability scheme given earlier, therefore it follows that asymmetric equilibria at two sexlinked loci should be relatively easy to determine.

Imposing the conditions $x_{2}=x_{3}=z$, but with $x_{1} \neq x_{4}$ on equation $(9 \mathrm{~b})$ gives

$$
\begin{aligned}
0= & 0+\frac{1}{2}\left(x_{1} z-z x_{4}\right)(1-\beta)\left(2-\delta_{\mathrm{m}}\right) \\
& +\frac{1}{2}\left(z x_{4}-x_{1} z\right)(1-\gamma)\left(2-\delta_{\mathrm{m}}\right)
\end{aligned}
$$

which is satisfied if $\beta=\gamma$ (Karlin and Feldman, 1970; Li, 1971). Proceeding using Li's (1971) method yields equilibrium solutions of the same form as the autosomal case namely,

$$
\begin{aligned}
& x_{1}=\frac{1}{2}[\xi-\sqrt{ } \hat{R}] \quad x_{1}=\frac{1}{2}[\xi+\sqrt{ } \hat{R}] \\
& x_{2}=x_{3}=\frac{1}{2}(1-\xi) \quad \text { and } \quad x_{2}=x_{3}=\frac{1}{2}(1-\xi) \\
& x_{4}=\frac{1}{2}[\xi+\sqrt{ } \hat{R}] \quad x_{4}=\frac{1}{2}[\xi-\sqrt{ } \hat{R}]
\end{aligned}
$$

(Karlin and Feldman, 1970) where, however,

$$
\xi=\frac{\beta-\frac{\alpha_{\mathrm{f}}}{2}+\frac{\delta_{\mathrm{m}}(1-\beta)}{2}+\frac{r_{\mathrm{f}} \delta_{\mathrm{f}}}{2\left(r_{\mathrm{f}}-\delta_{\mathrm{f}}\right)}}{2 \beta-\frac{\alpha_{\mathrm{f}}}{2}-\delta_{\mathrm{f}}-\delta_{\mathrm{m}}\left(\beta-\delta_{\mathrm{f}}\right)+\frac{r_{\mathrm{f}} \delta_{\mathrm{f}}}{2\left(r_{\mathrm{f}}-\delta_{\mathrm{f}}\right)}}
$$

and

$$
R=\xi^{2}-\left[r_{\mathrm{f}}(1-\xi)^{2} /\left(r_{\mathrm{f}}-\delta_{\mathrm{f}}\right)\left(1-\delta_{\mathrm{m}}\right)\right] .
$$

The necessary conditions for the existence of an equilibrium are therefore, $r_{\mathrm{f}}>\delta_{\mathrm{f}}, 0<\xi<1$ and

$$
\xi^{2} /(1-\xi)^{2}>r_{\mathrm{f}} /\left[\left(r_{\mathrm{f}}-\delta_{\mathrm{f}}\right)\left(1-\delta_{\mathrm{m}}\right)\right]>1 .
$$

Again when there is no selection in males the above equations reduce to the standard autosomal ones (Karlin and Feldman, 1970), with $r$ replaced by $r_{\mathrm{f}}$.

Proceeding heuristically to examine the stability of the equilibria, write

$$
\xi=\frac{(\beta-\theta)+\frac{1}{2} \delta_{\mathrm{m}}(1-\beta)}{\left(\beta-\delta_{\mathrm{f}}\right)\left(1-\delta_{\mathrm{m}}\right)+(\beta-\theta)}
$$


where

$$
\theta=\frac{\alpha_{\mathrm{f}}}{2}-\frac{r_{\mathrm{f}} \delta_{\mathrm{f}}}{2\left(r_{\mathrm{f}}-\delta_{\mathrm{f}}\right)} .
$$

For $\xi$ to be positive both numerator and denominator must be of the same sign, moreover it is known that for the autosomal case both must be negative for the equilibrium to be stable ( $\mathrm{Li}$, 1976). Assuming this to be true for the sex-linked case also, the following conditions are required: Numerator; $(\beta-\theta)+\frac{1}{2} \delta_{\mathrm{m}}(1-\beta)<0$ i.e. $\theta>$ $\beta+\frac{1}{2} \delta_{\mathrm{m}}(1-\beta)$. Denominator; First term, $\left(\beta-\delta_{\mathrm{f}}\right) \times$ $\left(1-\delta_{\mathrm{m}}\right)<0$, i.e. $\delta_{\mathrm{f}}>\beta$. Second term, $(\beta-\theta)<0$ i.e. $\theta>\beta$, which must be true if the first condition holds.

Now equation (19) implies that $\xi>\frac{1}{2}$ or $x_{1}+$ $x_{4}>x_{2}+x_{3}(\mathrm{Li}, 1971)$, therefore

$$
\frac{(\beta-\theta)+\frac{1}{2} \delta_{\mathrm{m}}(1-\beta)}{\left(\beta-\delta_{\mathrm{f}}\right)\left(1-\delta_{\mathrm{m}}\right)+(\beta-\theta)}>\frac{-1}{-2}
$$

is necessary (written this way because numerator and denominator must be negative), which gives, $\theta>\delta_{\mathrm{f}}\left(1-\delta_{\mathrm{m}}\right)+\delta_{\mathrm{m}}$. Therefore putting these together gives the condition for a stable equilibrium

$$
\theta>\delta_{\delta}\left(1-\delta_{\mathrm{m}}\right)+\delta_{\mathrm{m}}>\beta+\frac{1}{2} \delta_{\mathrm{m}}(1-\beta) .
$$

Note that if $\delta_{\mathrm{m}}=0$ then the inequality (21) reduces to $\theta>\delta_{\mathrm{f}}>\beta$, equivalent to the conditions for the autosomal case (Karlin and Feldman, 1970; Li, 1976).

Some examples of asymmetric equilibria are given in table 1 . The values of the female viability parameters chosen are the same as those of example II of Karlin and Feldman (1970); in their Table II. It can be seen that with symmetrical selection in males the gametic equilibrium values are shifted considerably and linkage disequili- brium increases. Also as $\delta_{\mathrm{m}}$ increases the conditions for the existence of a stable equilibrium (equations 19 and 21) become more stringent.

As in the autosomal case when $\beta \neq \gamma$ asymmetrical equilibria of the form $x_{1} \neq x_{4}, x_{2} \neq x_{3}$ occur, and an explicit solution could be found using the methods outlined by Karlin and Feldman (1970).

\section{(2) Male viabilities}

$$
v_{11}=v_{44}=1, \quad v_{22}=v_{33}=1-\alpha_{\mathrm{m}}
$$

\section{Symmetrical equilibria}

Symmetrical equilibria of the form $x_{1}=x_{4}, x_{2}=x_{3}$ will also occur with this type of symmetrical selection in males. Proceeding as before an equation analgogous to equation (10) is derived,

$$
\begin{gathered}
64 k D_{\mathrm{f}}^{3}-16\left[m-2 \alpha_{\mathrm{m}}\left(1+r_{\mathrm{f}}\right)\right] D_{\mathrm{f}}^{2} \\
-4\left[k-4 r_{\mathrm{f}}\left(2-\alpha_{\mathrm{m}}\right)\right] D_{\mathrm{f}} \\
+m-2 \alpha_{\mathrm{m}}\left(1-r_{\mathrm{f}}\right)=0
\end{gathered}
$$

where in this case,

$$
k=\left(2-\alpha_{\mathrm{m}}\right)(\beta+\gamma)-\left[\alpha_{\mathrm{f}}\left(1-\alpha_{\mathrm{m}}\right)+\delta_{\mathrm{f}}\right]
$$

and

$$
m=\delta_{\mathrm{f}}-\alpha_{\mathrm{f}}\left(1-\alpha_{\mathrm{m}}\right) .
$$

This can be solved for various special cases as was done previously, though this will not be done here.

\section{Asymmetrical equilibria}

Asymmetrical equilibria of the form $x_{1} \neq x_{4}, x_{2}=x_{3}$ (or vice versa) clearly exist with this type of symmetrical selection in males. The equilibrium gametic values are given by equations (16), where,

Table 1 Some examples of asymmetric equilibria at two sex-linked loci with symmetrical selection in females and males. The female viability parameters are; $\alpha_{\mathrm{f}}=0 \cdot 1, \gamma=\beta=0.005$, $\delta_{\mathrm{f}}=0.01$, and $r_{\mathrm{f}}=0.02$

1. $\delta_{\mathrm{m}}=0$

$x_{1}=0.0090$

$x_{2}=0.0625$

$x_{3}=0.0625$

$x_{4}=0.8660$

$D_{\mathrm{f}}=0.0039$

$y_{1}=0.0090$

$y_{2}=0.0625$

$y_{3}=0.0625$

$y_{4}=0.8660$

$D_{\mathrm{m}}=0.0039$

2. $\delta_{\mathrm{m}}=0.01$

$x_{1}=0.0441$

$x_{2}=0 \cdot 1242$

$x_{3}=0 \cdot 1242$

$x_{4}=0.7075$

$y_{1}=0.0440$

$y_{2}=0 \cdot 1251$

$y_{3}=0 \cdot 1251$

$y_{4}=0.7057$

$D_{\mathrm{f}}=0.0158$

$D_{\mathrm{m}}=0.0154$

3. $\delta_{\mathrm{m}}=0.015$

$x_{1}=0.0802$

$x_{2}=0 \cdot 1551$

$x_{3}=0 \cdot 1551$

$x_{4}=0.6095$

$D_{\mathrm{f}}=0.0248$

$y_{1}=0.0798$

$y_{2}=0.1567$

$y_{3}=0.1567$

$y_{4}=0.6067$

$D_{\mathrm{m}}=0.0239$ 
however in this case

$$
\begin{aligned}
\xi=\left[\beta\left(1-\frac{1}{2} \alpha_{\mathrm{m}}\right)-\left(\frac{\alpha_{\mathrm{f}}\left(1-\alpha_{\mathrm{m}}\right)}{2}\right)-\left(\frac{\alpha_{\mathrm{m}}}{2}\right)\right. \\
\left.+\left(\frac{r_{\mathrm{f}} \delta_{\mathrm{f}}\left(1-\alpha_{\mathrm{m}}\right)}{2\left(r_{\mathrm{f}}-\delta_{\mathrm{f}}\right)}\right)\right] /\left[2 \beta-\left(\frac{\alpha_{\mathrm{f}}}{2}\right)-\delta_{\mathrm{f}}\right. \\
\left.+\left(\frac{\left(2 \beta-\alpha_{\mathrm{f}}\right) \alpha_{\mathrm{m}}}{2}\right)+\left(\frac{r_{\mathrm{f}} \delta_{\mathrm{f}}\left(1-\alpha_{\mathrm{m}}\right)}{2\left(r_{\mathrm{f}}-\delta_{\mathrm{f}}\right)}\right)\right]
\end{aligned}
$$

and

$$
\hat{R}=\xi^{2}-\left[r_{\mathrm{f}}\left(1-\alpha_{\mathrm{m}}\right)(1-\xi)^{2} /\left(r_{\mathrm{f}}-\delta_{\mathrm{f}}\right)\right] .
$$

The conditions necessary for an equilibrium are, $r_{\mathrm{f}}>\delta_{\mathrm{f}}, 0<\xi<1$ and

$$
\xi^{2} /(1-\xi)^{2}>r_{\mathrm{f}}\left(1-\alpha_{\mathrm{m}}\right) /\left(r_{\mathrm{f}}-\delta_{\mathrm{f}}\right)>1 .
$$

\section{ASYMMETRICAL SELECTION IN MALES}

Consider selection with male viabilities

$$
v_{11}=1-s, \quad v_{22}=v_{33}=1, \quad v_{44}=1-t,
$$

and still assuming symmetrical selection in females. Obviously symmetrical equilibria of the form $x_{1}=x_{2}=x_{3}=x_{4}$ can no longer occur, in fact only asymmetrical equilibria of the type $x_{1} \neq x_{4}$, $x_{2}=x_{3}$ or $x_{1} \neq x_{4}, x_{2} \neq x_{3}$ are possible.

Substitution of the male viabilities given above and the symmetric female viabilities into equations (3) and subtraction of the second and third of these equations gives,

$$
\begin{aligned}
\hat{M}\left(x_{2}-x_{3}\right)= & \left(x_{2}^{2}-x_{3}^{2}\right)\left(1-\alpha_{\mathrm{f}}\right) \\
& +\frac{1}{2}\left[x_{1} x_{2}(2-s)-x_{3} x_{4}(2-t)\right](1-\beta) \\
& +\frac{1}{2}\left[x_{2} x_{4}(2-t)-x_{1} x_{3}(2-s)\right](1-\gamma) .
\end{aligned}
$$

Put $x_{2}=x_{3}=z$ then

$$
\begin{aligned}
0= & 0+\frac{1}{2}\left[x_{1} z(2-s)-z x_{4}(2-t)\right](1-\beta) \\
& +\frac{1}{2}\left[z x_{4}(2-t)-x_{1} z(2-s)\right](1-\gamma)
\end{aligned}
$$

which is satisfied if $\beta=\gamma$. Note, however, that equilibria of the form $x_{1}=x_{4}, x_{2} \neq x_{3}$ cannot occur.

Similar reasoning allows the type of equilibria possible under various asymmetrical selection schemes in males to be specified (table 2).

\section{THE HITCHHIKING EFFECT}

Another aspect of two-locus theory to receive attention is the effect that selection at one locus has on gene frequencies at a second linked, but neutral locus-this is the hitchhiking effect (Kojima and Lewontin, 1970; Maynard Smith and Haigh, 1974; Ohta and Kimura 1975; Thomson, 1977).

Sex-linked or haplodiploid loci are predicted to have a reduced level of heterozygosity as compared to autosomal loci, whether selection or mutation and random drift are maintaining most of the genetic variability (Lester and Selander, 1979; Pamilo and Crozier, 1981; Avery, 1984). This conclusion is based on single-locus theory only, therefore it is of interest to examine the effect of hitchhiking on genetic variation at sex-linked loci. There has been some discussion of the importance of hitchhiking in male haploids. Lester and Selander (1979) argue that hitchhiking should be stronger at sex-linked than at autosomal loci due to the effectively tighter linkage due to the lack of recombination in males. However Pamilo and Crozier (1981) point out that this argument does not apply to Drosophila which has no crossing over in males

Table 2 Types of equilibria possible when there is either symmetrical or asymmetrical selection in males superimposed on symmetrical selection in females at two sex-linked loci

Male Viabilities

\begin{tabular}{llll}
\hline$v_{11}$ & $v_{22}$ & $v_{33}$ & $v_{44}$ \\
\hline \multicolumn{2}{l}{ Symmetrical selection: } \\
$1-\delta_{\mathrm{m}} \quad 1$ & 1 & $1-\delta_{\mathrm{m}}$ \\
1 & $1-\alpha_{\mathrm{m}}$ & $1-\alpha_{\mathrm{m}}$ & 1
\end{tabular}

Asymmetrical selection:

$\begin{array}{llll}1-s & 1 & 1 & 1-t \\ 1 & 1-s & 1-t & 1 \\ 1-s & 1 & 1-t & 1 \\ 1 & 1-s & 1 & 1-t\end{array}$

\section{Equilibra}

$$
\beta=\gamma
$$

$\beta \neq \gamma$

$$
\begin{array}{r}
x_{1}=x_{2}=x_{3}=x_{4} \text { or } x_{1} \neq x_{4}, x_{2}=x_{3} \\
\text { or } x_{1}=x_{4}, x_{2} \neq x_{3} \\
x_{1}=x_{2}=x_{3}=x_{4} \\
\text { or } x_{1} \neq x_{4}, x_{2}=x_{3} \\
\text { or } x_{1}=x_{4}, x_{2} \neq x_{3}
\end{array}
$$

$$
\begin{aligned}
& x_{1}=x_{2}=x_{3}=x_{4} \text { or } x_{1} \neq x_{4}, x_{2} \neq x_{3} \\
& x_{1}=x_{2}=x_{3}=x_{4} \text { or } x_{1} \neq x_{4}, x_{2} \neq x_{3}
\end{aligned}
$$

$\begin{array}{ll}x_{1} \neq x_{4}, x_{2}=x_{3} & x_{1} \neq x_{4}, x_{2} \neq x_{3} \\ x_{1}=x_{4}, x_{2} \neq x_{3} & x_{1} \neq x_{4}, x_{2} \neq x_{3} \\ x_{1} \neq x_{4}, x_{2} \neq x_{3} & x_{1} \neq x_{4}, x_{2} \neq x_{3} \\ x_{1} \neq x_{4}, x_{2} \neq x_{3} & x_{1} \neq x_{4}, x_{2} \neq x_{3}\end{array}$


yet is highly heterozygous. Nevertheless they note that because with weak, directional selection alleles are substituted more rapidly at sex-linked than at autosomal loci (Avery, 1984), linked neutral alleles will also be carried to fixation at the same rate. Thus hitchhiking potentially could be more effective at sex-linked loci as compared to autosomal loci. In order to quantify these arguments equations analogous to those of Kojima and Lewontin (1970) and Thomson (1977) are now derived for the case of weak selection at sex-linked loci.

Consider two loci, $B$ neutral and $A$ selected with fitnesses:

\begin{tabular}{ccccccc}
\multicolumn{3}{c}{ Females } & & \multicolumn{3}{c}{ Males } \\
\cline { 1 - 2 } \cline { 5 - 6 } & $A a$ & $a a$ & & $A$ & $a$ \\
$1-s$ & $1-h s$ & 1 & & $1-s$ & 1
\end{tabular}

Let $p_{A}, p_{a}, p_{B}, p_{b}$ be the frequencies of alleles $A$, $a, B, b$ respectively in females and $P_{A}, P_{a}, P_{B}, P_{b}$ their frequencies in males. Assuming weak selection (i.e., $s$ is reasonably small) then $P_{A} \cong p_{A}, P_{B} \cong$ $p_{B}, \bar{w} \cong \bar{v} \cong 1, D_{\mathrm{f}} \cong D_{\mathrm{m}}=D$ and $x_{i} \cong y_{i}$. Then, using primes to denote values in the next generation, equations (1) and (2) become,

Females:

$$
\left.\begin{array}{l}
x_{1}^{\prime} \cong x_{1}\left[1-s\left(p_{A}+p_{a} h\right)\right]-r_{\mathrm{f}} D \\
x_{2}^{\prime} \cong x_{2}\left[1-s\left(p_{A}+p_{a} h\right)\right]+r_{\mathrm{f}} D \\
x_{3}^{\prime} \cong x_{3}\left[1-s h p_{A}\right]+r_{\mathrm{f}} D \\
x_{4}^{\prime} \cong x_{4}\left[1-s h p_{A}\right]-r_{\mathrm{f}} D .
\end{array}\right\}
$$

Males:

$$
\left.\begin{array}{l}
y_{1}^{\prime} \cong x_{1}(1-s) \\
y_{2}^{\prime} \cong x_{2}(1-s) \\
y_{3}^{\prime} \cong x_{3} \\
y_{4}^{\prime} \cong x_{4} .
\end{array}\right\}
$$

The change in average gene frequency at locus $B$ is approximately (Avery, 1984),

$$
\begin{aligned}
\Delta \bar{p}_{B} & =\left[\left(2 p_{B}^{\prime}+P_{B}^{\prime}\right)-\left(2 p_{B}+P_{B}\right)\right] / 3 \\
& \cong-\frac{s}{3}\left[(1+2 h)+(1-2 h) 2 p_{A}\right] D .
\end{aligned}
$$

Therefore

$$
\Delta \bar{p}_{B} \cong \frac{\Delta \bar{p}_{A}}{p_{A} p_{a}} \cdot D
$$

where

$$
\Delta \vec{p}_{A} \cong-\frac{s}{3} p_{A} p_{a}\left[(1+2 h)+(1-2 h) 2 p_{A}\right],
$$

which, of course, is the familiar equation for gene frequency change under weak selection (Nagylaki, 1979; Avery, 1984). It also follows that the amount of linkage disequilibrium between the two loci is changing each generation according to the relationship,

$$
D^{\prime} \cong\left[\frac{p_{A}^{\prime} p_{a}^{\prime}}{p_{A} p_{a}}-(1-h s) r_{\mathrm{f}}\right] D .
$$

This formula is equivalent to Thomson's (1977) equation (28) for, of course, the case of weak selection. Therefore $D$ can be expressed as (Thomson, 1977),

$$
\begin{aligned}
D(n+1) & \cong A_{n} D(n) \\
& \cong B_{n+1} D(o)
\end{aligned}
$$

where

$$
B_{0}=1 \quad \text { and } \quad B_{n}=\prod_{i=0}^{n-1} A_{i}
$$

with

$$
A_{n}=\frac{p_{A}(n+1) p_{a}(n+1)}{p_{A}(n) p_{a}(n)}-(1-h s) r_{\mathrm{f}} .
$$

The case considered here is that of a new mutant allele $a$, which eventually goes to fixation under directional selection. Thomson (1977) has shown that after selection at locus $A$, the mean heterozyosity at locus $B$ is,

$$
\bar{H}=2\left(1-E^{2}\right) p_{B}(o) p_{b}(o)
$$

where, for the sex-linked case,

$$
E \cong \sum_{k=0}^{n} \frac{\Delta \bar{p}_{a}(k)}{p_{A}(k) p_{a}(k)} B_{k} p_{a}(o)
$$

Thus mean heterozygosity is reduced to $\left(1-E^{2}\right)$ times its initial value. Clearly, as is apparent from equation (35), the value of $E$ depends on both the magnitude of $\Delta \bar{p}_{a}$ per generation and the number of generations over which gene frequency change occurs. Now although the rate of gene frequency change, at a given gene frequency, is greater at $\mathrm{X}$-linked (equation (32)) than autosomal loci (Nagylaki, 1979; Avery, 1984), this also means that the number of generations until fixation is greater at autosomal loci, giving the hitchhiking effect a longer time over which to operate. Hence the hitchhiking effect is not necessarily expected to be stronger at X-linked loci. 
The reduction in heterozygosity at $\mathrm{X}$-linked and autosomal loci caused by the hitchhiking effect was compared by iterating the two locus recurrence equations for various combinatins of $s, h$ and the recombination fraction (table 3 ). Two situations were looked at; the first being the Drosophila type where at autosomal, as well as at sex-linked, loci there is no recombination in males, and the second being the more common type where recombination occurs in both sexes at autosomal loci. Equations (1) and (2) were iterated for the sex-linked case, and the equations of Strobeck (1974) were iterated for the autosomal case. Initial heterozygosity at the neutral locus $(B)$ was $H_{o}=2 p_{B}(o) p_{b}(o)=0 \cdot 5$, and selection at locus $A$ was carried out from $p_{a}(o)=0 \cdot 01$ to $p_{a}(t)=0.99$. Table 3 gives the ratio of the final mean heterozygosity at the X-linked locus to that at the autosomal locus $(\bar{H} x(t) / \bar{H} a(t))$. Thomson (1977) has pointed out that iterations starting from $H_{o}=0.5$ give the average reduction in heterozygosity. Some interesting conclusions can be drawn from the results given in table 3. Clearly in organisms such as Drosophila which have no recombination in males, the hitchhiking effect can be stronger at autosomal loci, but almost without exception only when the selected allele is dominant $(h=0)$. If it is codominant $(h=$ $\frac{1}{2}$ ) then, unless $s=0 \cdot 5$, there is essentially no difference between $\mathrm{X}$-linked and autosomal loci in the degree to which heterozygosity is reduced. On the other hand if the selected allele is recessive $(h=1)$ then X-linked loci always undergo an often considerable reduction in heterozygosity compared to autosomal loci. This is because hitchiking is relatively ineffective at autosomal loci in this case (Maynard Smith and Haigh, 1974), and therefore the effect at X-linked loci becomes relatively pronounced. The situation is quite different when there is recombination in males at autosomal loci. Now the hitchhiking effect is always stronger at the X-linked loci (for, of course, $s \geqq r$, over which the effect operates). However note that in the case of a selected recessive allele the relative reduction in heterozygosity is no greater than when there is no recombination in males at their autosomal loci, at least when $s \leqq 0 \cdot 05$. This is again a reflection of the inefficiency of hitchhiking at autosomal loci when $h=1$.

Hitchhiking has been treated here purely deterministically, so it must be remembered that with weak selection genetic drift will overpower hitchhiking in small populations. However hitchhiking will predominate even with $s=0.005$ if population size is sufficiently large, i.e. $>10^{5}$ (Thomson, 1977).

Table 3 Ratio of final mean heterozygosity at a neutral sex-linked locus to that at a neutral autosomal locus, each linked to locus which has undergone selection. Two cases are compared. I, no recombination in males at autosomal loci (Drosophila) II, recombination in males at autosomal loci

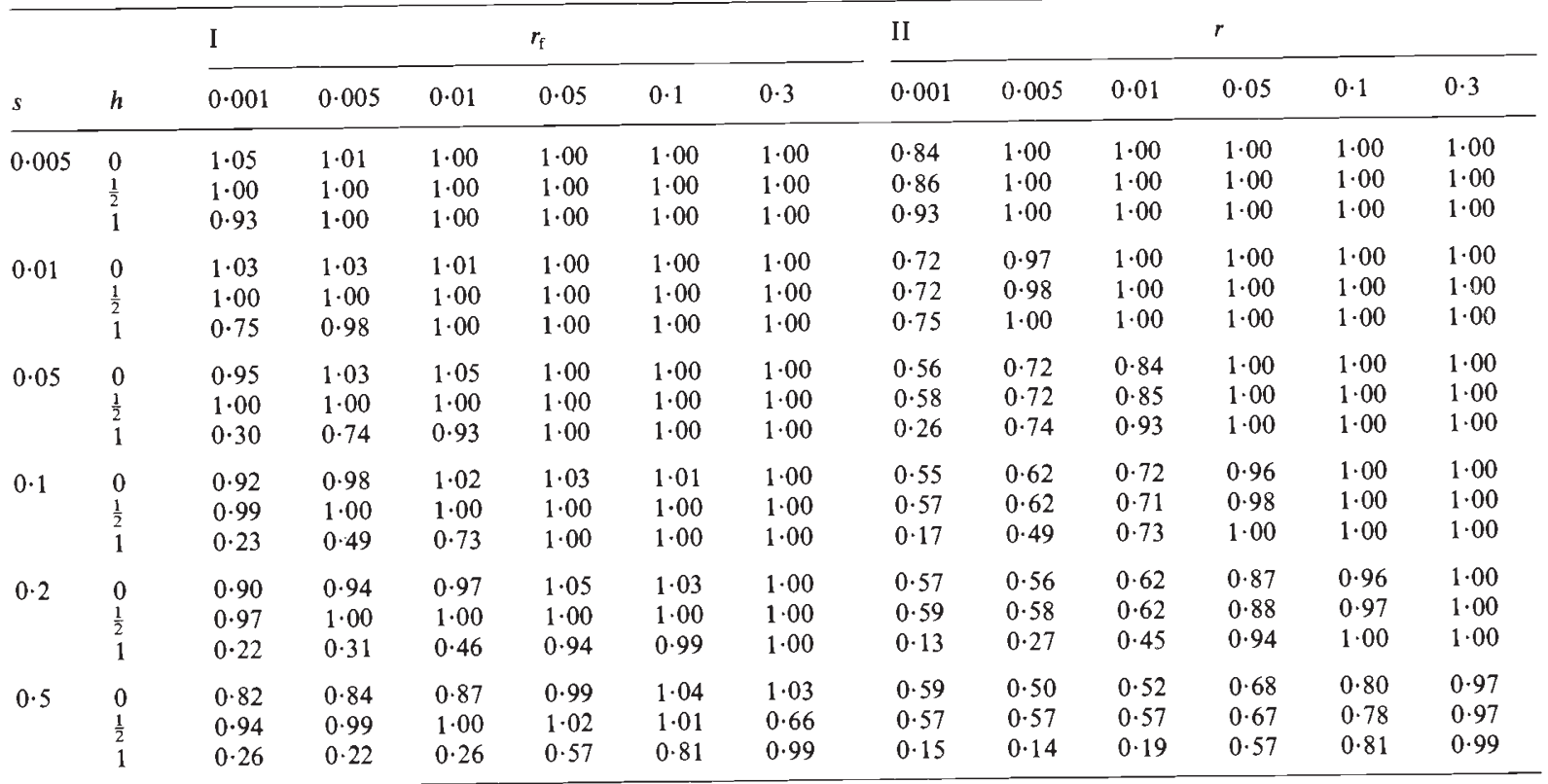




\section{DISCUSSION}

Selection at two sex-linked loci is in some ways a combination of selection at autosomal and haploid loci, nevertheless as such it possesses some unique features. One of the most interesting is that gametic equilibria occur only at a stationary point of the mean viability if

$$
\frac{1}{2} r_{\mathrm{f}}\left[x_{\mathrm{I}} x_{4}\left(v_{11}+v_{44}\right)-x_{2} x_{3}\left(v_{22}+v_{33}\right)\right] w_{22}=0,
$$

which is a more stringent condition than the autosomal equivalent, $r D w_{22}=0$. The stationary point, of course, may or may not be a maximum and certainly mean fitness will not necessarily increase under selection; even at a single sex-linked locus geometric mean viability is not an increasing function under selection (Cannings, 1969b; Edwards, 1977). However the important result is that linkage equilibrium, in either one or both sexes will not occur even if mean fitness is maximized at gametic equilibrium.

The autosomal two locus symmetrical selection model has been analysed extensively, and it can be easily modified for the case of sex-linkage to incorporate simple symmetrical selection in males, allowing for a total of five viability parameters, four in females and one in males. As with autosomal loci both symmetrical and asymmetrical equilibria can occur, and analogous equations for $D_{\mathrm{f}}$ (coefficient of linkage disequilibrium in females) and the gametic equilibrium frequencies can easily be derived. However, even with simple symmetrical selection in males the equations are considerably more complicated than the equivalent autosomal ones. Stability of the symmetrical equilibria was not examined, but the rather heuristic analysis suggested that the conditions for stability of asymmetrical equilibria are more stringent at sex-linked than autosomal loci. Intuitively this seems reasonable since, as is well known, the conditions for a balanced polymorphism at a single locus are more severe in the sexlinked case.

Asymmetrical selection in males removes all possibility of having symmetrical equilibria $\left(x_{1}=\right.$ $\left.x_{2}=x_{3}=x_{4}\right)$, therefore even with symmetrical selection in females, the outcome of selection at two sex-linked loci is very sensitive to selection in the haploid males. Unless there is a particular balance of selection coefficients in males asymmetrical equilibria are bound to result. Another interesting consequence of selection at two sexlinked loci is that some linkage disequilibrium is bound to result, either in the females or the males or both.
Finally it was shown that the reduction in heterozygosity at a neutral locus caused by selection at a linked locus (the hitchhiking effect) is not necessarily greater at sex-linked loci than at autosomal loci. If there is no recombination in males at the autosomal loci (the situation in Drosophila), then with relatively weak selection and dominance of the selected allele, a greater reduction in heterozygosity occurs than at a corresponding sexlinked locus.

Acknowledgment The support of the Natural Sciences and Engineering Research Council of Canada is gratefully acknowledged.

\section{REFERENCES}

ARUnACHAlam, V. AND OWEN, A. R. G. 1971. Polymorphisms with Linked Loci. Chapman and Hall, London.

AVERY, P. J. 1984. The population genetics of haplodiploids and X-linked genes. Genet. Res., 44, 321-341.

BENNETT, J. H. 1963. Random mating and sex linkage. J. Theor. Biol., 4, 28-36.

BODMER, W. F. AND FELSENSTEIN, J. 1967. Linkage and selection: theoretical analysis of the deterministic two locus random mating model. Genetics, 57, 237-265.

CANNINGS, C. 1968. Equilibrium under selection at a multiallelic sex-linked locus. Biometrics, 24, 187-189.

CANNINGS, C. 1969a. The study of multiallelic genetic systems by matrix methods. Genet. Res., 14, 167-183.

CANNINGS, C. $1969 \mathrm{~b}$. A note on stability and convergence of genetic systems. Evolution, 23, 517-518.

CLEGG, M. T. AND CAVENER, D. R. 1982. Dynamics of correlated genetic systems. VII. Demographic aspects of sexlinked transmission. Amer. Nat.. 120.108-118.

EDWARDS, A. W. F. 1977. Foundations of Mathematical Genetics. Cambridge University Press, Cambridge.

EWENS, W. J. 1979. Mathematical Population Genetics. SpringerVerlag, Berlin.

FELDMAN, M. W. 1971. Equilibrium studies of two locus haploid populations with recombination. Theor. Pop. Biol., 2, 299-318.

KARLIN, S. 1975. General two-locus selection models: some objectives, results and interpretations. Theor. Pop. Biol., 7, 364-398.

KARLIN, S. AND FELDMAN, M. W. 1970. Linkage and selection: two locus symmetric viability model. Theor. Pop. Biol., 1, 39-71.

KIMURA, M. 1956. A model of a genetic system which leads to closer linkage by natural selection. Evolution, 10, 278-287.

KOJIMA, K. AND LEWONTIN, R. C. 1970. Evolutionary significance of linkage and epistasis. In Kojima, K. (ed.) Topics in Mathematical Genetics, Springer-Verlag, New York.

LESTER, L. J. AND SELANDER, R. K. 1979. Population genetics of haplodiploid insects. Genetics, 92, 1329-1345.

LEWONTIN, R. C. AND KOJIMA, K. 1960. The evolutionary dynamics of complex polymorphisms. Evolution, 14, 458472.

Lr, C. C. 1967 . The maximization of average fitness by natural selection for a sex-linked locus. Proc. Nat. Acad. Sci. U.S.A., $57,1260-1261$ 
LI, C. C. 1971. Unsymmetric equilibria under two-locus symmetric selection model. J. Hered., 62, 47-48.

LI, C. C. 1976. First Course in Population Genetics. Boxwood Press, Pacific Grove, California.

MAYNARD SMITH, J. AND HAIGH, J. 1974. The hitchhiking effect of a favourable gene. Genet. Res., 23, 23-35.

MORAN, P. A. P. 1964. On the nonexistence of adaptive topographies. Ann. Hum. Genet, 27, 383-393.

NAGYLAKI, T. 1977. Selection in One-and Two-Locus Systems, Lecture Notes in Biomathematics, 15. Springer-Verlag, Berlin.

NAGYlaki, T. 1979. Selection in dioecious populations. Ann. Hum. Genet., 43, 143-150.

NEI, M. 1967. Modification of linkage intensity by natural selection. Genetics, 57, 625-641.
OHTA, T. AND KIMURA, M. 1975. The effect of selected linked locus on heterozygosity of neutral alleles (the hitchhiking effect). Genet. Res., 25, 313-326.

OWEN, R. E. 1980. Population genetics of social Hymenoptera with worker produced males. Heredity, 45, 31-46.

PAMILO, P. AND CROZIER, R. H. 1981. Genic variation in male haploids under deterministic selection. Genetics, 98, 199214.

STROBECK, C. 1974. The two-locus model with sex differences in recombination. Genetics, 78, 791-797.

THOMSON, G. 1977. The effect of a selected locus on linked neutral loci. Genetics, 85, 753-788.

WRIGHT, S. 1933. Inbreeding and recombination. Proc. Nat. Acad. Sci. U.S.A., 19, 420-433. 